\title{
Diffractive Neutral Pion Production, Chiral Symmetry and the Odderon
}

\author{
Carlo Ewerz ${ }^{a}$, Otto Nachtmann ${ }^{b \dagger}$ \\ ${ }^{a}$ ECT*$^{*}$, Strada delle Tabarelle 286, I-38050 Villazzano (Trento), Italy \\ ${ }^{b}$ Institut für Theoretische Physik, Universität Heidelberg, Philosophenweg 16, \\ D-69120 Heidelberg, Germany
}

\begin{abstract}
We discuss the diffractive photo- and electroproduction of a single neutral pion at high energies where it can occur due to odderon exchange. We show that this process is dynamically suppressed as a consequence of chiral symmetry. Our result reconciles earlier theoretical expectations with the non-observation of this reaction at HERA.
\end{abstract}

\section{Diffractive neutral pion production as a probe of the odderon}

In this talk we want to present the main results of our study [1] of diffractive production of a single neutral pion in photon-proton scattering at high energy,

$$
\gamma^{(*)}(q)+p(p) \longrightarrow \pi^{0}\left(q^{\prime}\right)+X\left(p^{\prime}\right),
$$

where the photon can be real or virtual, and $X$ can be any diffractively produced hadronic system. For simplicity we will in the following assume that $X$ is a proton, but our considerations can also be applied to other states $X$, for example for $X=N^{*}$ or $X=n+\pi^{+}$[1]. Since the photon and the neutral pion have opposite $C$-parity the object exchanged in this reaction must be odd under charge conjugation, and hence at high energy must be an odderon $(\mathbb{O})$, see Figure 1. (Note that we draw the incoming particles to the right.)

The odderon, the $C=-1$ partner of the pomeron, was introduced in [2], for a general review see [3]. It has since been studied in great detail especially from a theoretical point of view. But experimentally the odderon remains an elusive object. Some weak evidence for its existence has only been seen in elastic scattering at the ISR where the $p p$ and $p \bar{p}$ differential cross sections show a difference at around $|t| \sim 1.3 \mathrm{GeV}^{2}$ [4], for a recent discussion see [5]. There, however, the odderon is only one among many contributions and hence difficult to pin down. In recent years it has been realized that the chances to observe the odderon are better in exclusive processes in which the odderon essentially gives the only contribution. As an important example of this strategy the reaction (1) has been proposed and discussed in [6].

A detailed analysis based on a nonperturbative model of QCD dynamics performed in [7] led to the prediction

$$
\sigma\left(\gamma p \rightarrow \pi^{0} N^{*}\right) \approx 300 \mathrm{nb},
$$

while the subsequent experimental search at HERA [8] did not find a signal and resulted in the upper bound

$$
\sigma\left(\gamma p \rightarrow \pi^{0} N^{*}\right)<49 \mathrm{nb} .
$$

\footnotetext{
${ }^{\dagger}$ speaker
} 


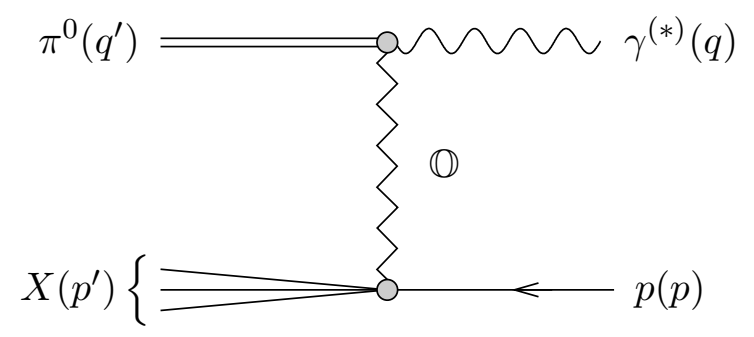

Fig. 1: Diffractive photo- or electroproduction of a $\pi^{0}$ due to odderon exchange.

Possible causes for the failure of the prediction of [7] were discussed in [9]. Since the reaction (1) has the largest phase space of all processes in which hadrons are diffractively produced a strong dynamical suppression appeared necessary in order to provide a likely reason. In [1] we have found that the approximate chiral symmetry of QCD indeed induces a strong suppression.

\section{PCAC}

Let us define a quark field operator describing up and down type quarks, $\psi(x)=(u(x), d(x))^{\mathrm{T}}$, and the associated triplet of axial vector currents $(a=1,2,3)$

$$
A_{\mu}^{a}(x)=\bar{\psi}(x) \gamma_{\mu} \gamma_{5} \frac{\tau^{a}}{2} \psi(x) .
$$

The well-known PCAC relation states that the divergence of this axial vector current is related to a correctly normalised pion field operator $\phi^{a}$ by

$$
\partial_{\lambda} A^{a \lambda}(x)=\frac{f_{\pi} m_{\pi}^{2}}{\sqrt{2}} \phi^{a}(x),
$$

where $f_{\pi} \approx 130 \mathrm{MeV}$ is the pion decay constant, see for example [10]. Let us now consider along with diffractive pion production the corresponding production of an axial vector current $A^{3}$,

$$
\begin{aligned}
& \gamma^{(*)}(q, \nu)+p(p, s) \quad \longrightarrow \quad \pi^{0}\left(q^{\prime}\right)+p\left(p^{\prime}, s^{\prime}\right), \\
& \gamma^{(*)}(q, \nu)+p(p, s) \quad \longrightarrow \quad A^{3}\left(q^{\prime}, \mu\right)+p\left(p^{\prime}, s^{\prime}\right),
\end{aligned}
$$

and let us denote the corresponding amplitudes by $\mathcal{M}^{\nu}\left(\pi^{0} ; q^{\prime}, p, q\right)$ and $\mathcal{M}^{\mu \nu}\left(A^{3} ; q^{\prime}, p, q\right)$, respectively, which we consider for $q^{2} \leq 0$ and $q^{\prime 2} \leq m_{\pi}^{2}$. Using the PCAC relation (5) we can then express the former amplitude in terms of the latter via

$$
\mathcal{M}^{\nu}\left(\pi^{0} ; q^{\prime}, p, q\right)=\frac{2 \pi m_{p} \sqrt{2}}{f_{\pi} m_{\pi}^{2}}\left(-q^{\prime 2}+m_{\pi}^{2}\right) i q_{\mu}^{\prime} \mathcal{M}^{\mu \nu}\left(A^{3} ; q^{\prime}, p, q\right) .
$$

\section{Axial vector current production}

The amplitude $\mathcal{M}^{\mu \nu}\left(A^{3} ; q^{\prime}, p, q\right)$ for axial vector current production (7) can be treated with the same general nonperturbative methods that were developed for Compton scattering in [11]. We 
(a)

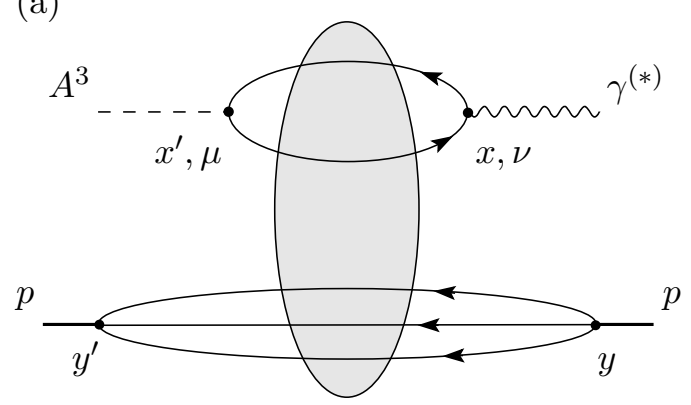

(b)

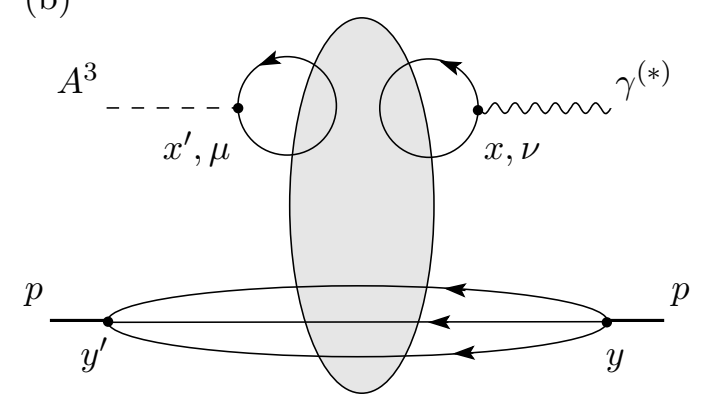

Fig. 2: Leading diagrams at high energies for the reaction (7).

use the LSZ formula to relate the amplitude to Green's functions, and the latter can then be written as functional integrals over quark and gluon fields. Integrating out the quark degrees of freedom leads us to diagram classes characterised by their quark line skeleton. Those diagrams which contain the leading terms at high energies are shown in Figure 2, and they are exactly the odderon exchange diagrams on which we want to concentrate here. The solid lines represent full quark propagators in a given gluon field configuration, and the shaded blobs indicate the functional integral over the gluon fields.

\section{From axial vector current to pion production}

We now consider the divergence of the amplitudes of Figure 2, that is we contract them with $q^{\prime \mu}$ or take a derivative $\partial^{\mu}$. For the axial vector current this gives at the quark level

$$
\partial^{\mu} A_{\mu}^{3}(x)=i\left[m_{u} \bar{u}(x) \gamma_{5} u(x)-m_{d} \bar{d}(x) \gamma_{5} d(x)\right],
$$

and hence there is a factor $m_{q}$ of the light quark masses in the divergence amplitude. Note that the gluon anomaly does not contribute here. Such anomalous pieces are contained in the individual contribution of the quark flavours to the divergence of $A_{\mu}^{3}$ and would have the quark line topology of diagram (b) in Figure 2, but they cancel in $\partial^{\mu} A_{\mu}^{3}$.

The quark loops in the diagrams of Figure 2 which couple to the axial vector current contain a factor $\gamma_{5}$. As a consequence, these loops give rise to an additional factor $m_{q}$ of the light quark mass. This can be shown in a more detailed analysis which makes use of LippmannSchwinger equations for the quark propagator, for details see [1]. Hence we find that the divergence amplitude $q_{\mu}^{\prime} \mathcal{M}^{\mu \nu}\left(A^{3} ; q^{\prime}, p, q\right)$ is proportional to the square of the light quark masses. More precisely,

$$
q_{\mu}^{\prime} \mathcal{M}^{\mu \nu}\left(A^{3} ; q^{\prime}, p, q\right)=m_{u}^{2} \mathcal{C}^{(u) \nu}\left(q^{\prime}, p, q\right)-m_{d}^{2} \mathcal{C}^{(d) \nu}\left(q^{\prime}, p, q\right)
$$

where the functions $\mathcal{C}^{(q) \nu}$ have pion poles but are otherwise finite. (These poles are cancelled by the explicit factor $\left(-q^{2}+m_{\pi}^{2}\right)$ in (8) when we insert (10) there.) We know from the theory of chiral symmetry that the squared pion mass is linear in the light quark masses,

$$
m_{\pi}^{2}=B\left(m_{u}+m_{d}\right)
$$


with

$$
B=-\frac{2}{f_{\pi}^{2}}\langle 0|\bar{u}(x) u(x)| 0\rangle \approx 1800 \mathrm{MeV} .
$$

Therefore we can conclude from (8) and (10) that the odderon exchange amplitude for pion production is proportional to the square of the pion mass,

$$
\mathcal{M}^{\nu}\left(\pi^{0} ; q^{\prime}, p, q\right) \sim \frac{1}{m_{\pi}^{2}} \mathcal{M}^{\mu \nu}\left(A^{3} ; q^{\prime}, p, q\right) \sim \frac{1}{m_{\pi}^{2}} m_{q}^{2} \sim m_{\pi}^{2} .
$$

We thus find that the odderon exchange amplitude for $\pi^{0}$-production vanishes in the chiral limit $m_{\pi}^{2} \rightarrow 0$. This result can be generalised to the reaction (1) with an arbitrary hadronic final state $X$.

\section{Conclusion}

We have considered the diffractive process $\gamma^{(*)} p \rightarrow \pi^{0} X$ at high energies where it should be dominated by odderon exchange. As a consequence of chiral symmetry the odderon exchange amplitude for this process vanishes in the chiral limit $m_{\pi}^{2} \rightarrow 0$. We still expect a strong dynamical suppression in the case of approximate chiral symmetry as it is realised in Nature. The cross section should be suppressed by a factor $m_{\pi}^{4} / M^{4}$, where $M$ is a mass scale characterising the scattering process. In the calculation of the process $\gamma p \rightarrow \pi^{0} N^{*}$ in [7] that effect had not been properly taken into account. A numerical estimate suggests that due to chiral symmetry the cross section found there is reduced by a factor of at least about 50 [9], changing the prediction (2) to less than about $6 \mathrm{nb}$. That reconciles the theoretical expectation with the experimental upper bound (3) of [8].

The considerations that we have outlined here can also be applied to pion production in other diffractive processes. An example that is relevant at the LHC and a future ILC is the quasidiffractive reaction $\gamma \gamma \rightarrow \pi^{0} \pi^{0}$ at high energies. Also this reaction is at high energies mediated by odderon exchange. Here an even stronger suppression due to approximate chiral symmetry is expected.

\section{References}

[1] C. Ewerz and O. Nachtmann, Eur. Phys. J. C49, 685 (2007). hep-ph/ 0608082.

[2] L. Lukaszuk and B. Nicolescu, Nuovo Cim. Lett. 8, 405 (1973);

D. Joynson, E. Leader, B. Nicolescu, and C. Lopez, Nuovo Cim. A30, 345 (1975).

[3] C. Ewerz (2003). hep-ph/0306137.

[4] A. Breakstone et al., Phys. Rev. Lett. 54, 2180 (1985).

[5] H. G. Dosch, C. Ewerz, and V. Schatz, Eur. Phys. J. C24, 561 (2002). hep-ph/ 0201294.

[6] A. Schäfer, L. Mankiewicz, and O. Nachtmann. In Proc. of the Workshop Physics at HERA, DESY Hamburg 1991, vol. 1, p. 243;

V. V. Barakhovsky, I. R. Zhitnitsky, and A. N. Shelkovenko, Phys. Lett. B267, 532 (1991);

W. Kilian and O. Nachtmann, Eur. Phys. J. C5, 317 (1998). hep-ph/ 9712371.

[7] E. R. Berger et al., Eur. Phys. J. C9, 491 (1999). hep-ph/9901376.

[8] H1 Collaboration, C. Adloff et al., Phys. Lett. B544, 35 (2002). hep-ex/ 0206073.

[9] A. Donnachie, H. G. Dosch, and O. Nachtmann, Eur. Phys. J. C45, 771 (2006). hep-ph/ 0508196. 
[10] S. L. Adler and R. F. Dashen, Current Algebras and Applications to Particle Physics. Benjamin, New York, 1968.

[11] C. Ewerz and O. Nachtmann, Annals Phys. 322, 1635 (2007). hep-ph/ 0404254 ; C. Ewerz and O. Nachtmann, Annals Phys. 322, 1670 (2007). hep-ph/ 0604087. 